\title{
Exploring the connection between stellar halo profiles and accretion histories in $L_{*}$ galaxies
}

\author{
Nicola C. Amorisco ${ }^{1,2}$ \\ ${ }^{1}$ Institute for Theory and Computation, Harvard University, 60 Garden Street, Cambridge, \\ MA 02138, USA \\ email: nicola.amorisco@cfa.harvard.edu \\ ${ }^{2}$ Max Planck Institute for Astrophysics, Karl-Schwarzschild-Strasse 1, 85748 Garching, \\ Germany
}

\begin{abstract}
I use a library of controlled minor merger N-body simulations, a particle tagging technique and Monte Carlo generated $\Lambda \mathrm{CDM}$ accretion histories to study the highly stochastic process of stellar deposition onto the accreted stellar halos (ASHs) of $L_{*}$ galaxies. I explore the main physical mechanisms that drive the connection between the accretion history and the density profile of the ASH. I find that: i) through dynamical friction, more massive satellites are more effective at delivering their stars deeper into the host; ii) as a consequence, ASHs feature a negative gradient between radius and the local mass-weighed virial satellite-to-host mass ratio; iii) in $L_{*}$ galaxies, most ASHs feature a density profile that steepens towards sharper logarithmic slopes at increasing radii, though with significant halo-to-halo scatter; iv) the ASHs with the largest total ex-situ mass are such because of the chance accretion of a small number of massive satellites (rather than of a large number of low-mass ones).
\end{abstract}

Keywords. galaxies: halos, galaxies: kinematics and dynamics, galaxies: structure, galaxies: interactions, galaxies: evolution

\section{Introduction}

The accreted stellar halo (ASH) of a galaxy represents a record of the accretion history of the galaxy itself. Its assembly is determined by a large number of free parameters, including the structural properties of each accreted satellite (virial mass, concentration, stellar content, morphology), the orbital properties of each accretion event (energy and angular momentum at infall), the structural properties of the host itself during accretion. This implies a significant degree of stochasticity, as shown by the observed halo-to-halo scatter (e.g., van Dokkum et al. 2014) and by the dichotomy between the 'broken' and sharply declining density profile of the stellar halo of the Milky Way (e.g., Deason et al. 2013) and the more extended halo of Andromeda, whose density profile is well described by a single power-law (e.g., Gilbert et al. 2012, Ibata et al. 2014).

\section{Individual contributions to the accreted stellar halo}

In Amorisco (2015) I have isolated the main ingredients that shape the contribution of each accreted satellite to the ASH. I adopted a simplified approach and assumed that the contributing dwarfs are dark matter dominated (as expected for the case of an $L_{*}$ host) and ignored the gravitational influence of the host's disk. Combined with the halo mass-concentration relation (e.g., Gao et al. 2008, Ludlow et al. 2014), this reduces the structural properties of each minor merger to two dimensionless parameters. 
a)
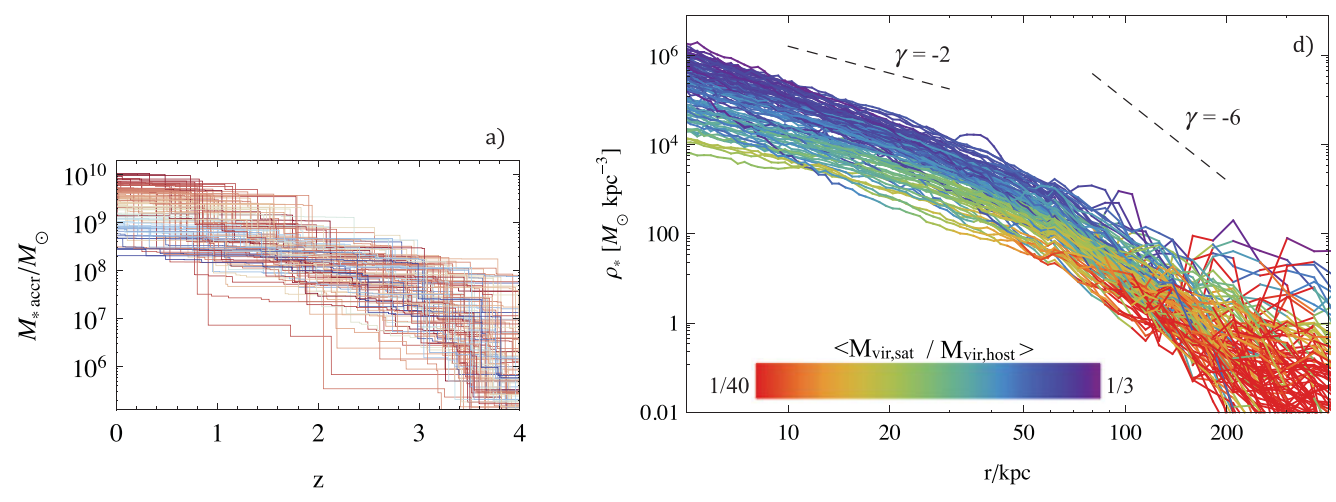

b)
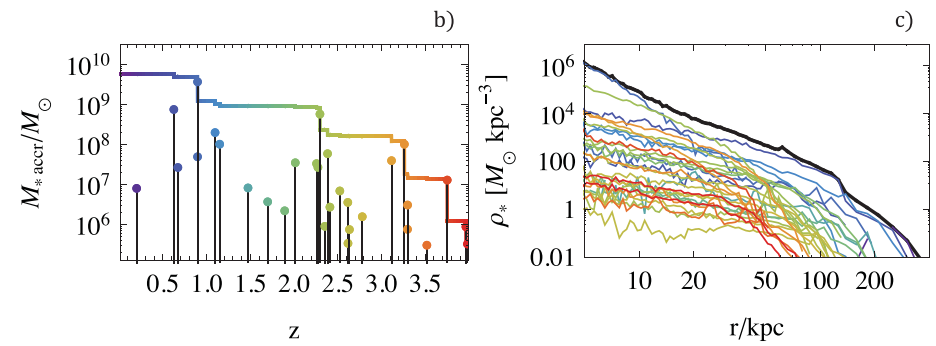

c)

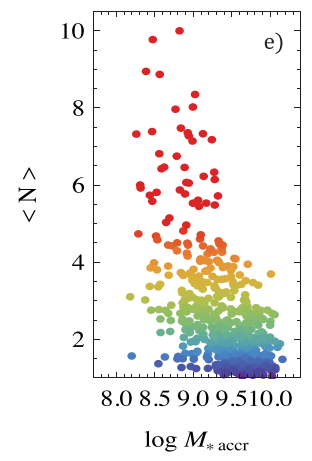

Figure 1. Panel a): a sample of 150 Monte Carlo generated stellar accretion histories for $L_{*}$ galaxies sharing a final virial mass of $2 \times 10^{12} M_{\odot}$. Panels b) and c): an example for the procedure of assembly of the accreted stellar halo using the combination of a given accretion history and of a library of minor merger simulations; each contribution to the halo is color-coded by accretion redshift. Panel c): the spherically averaged density profiles of the 150 accreted stellar haloes corresponding to the accretion histories of panel a), color-coding refers to the local mass-weighed virial satellite-to-host mass. Panel e): the correlation between the total accreted stellar mass in the halo and the number of main accretion events (see text for details).

Two additional parameters characterise the orbital properties of the satellite at accretion (e.g., Benson 2005, Jiang et al. 2015). The locus of this parameter space that is relevant to a $\Lambda$ CDM cosmology is explored with a library of minor merger N-body simulations, in which stars are assigned to the most bound $5 \%$ of the satellite's particles, using a standard particle tagging technique (e.g., Bullock \& Johnston 2005, Cooper et al. 2010). This study shows that dynamical friction is a major player in shaping stellar deposition, allowing only the most massive (and/or concentrated) accreted satellites to deposit their stars in the innermost regions of the host. Orbital radialisation by dynamical friction causes the stellar populations deposited by such most massive accretion events (virial satellite-to-host mass ratio at accretion $\gtrsim 1 / 20$ ) to bear little memory of the details of the orbital properties of the progenitor at infall.

\section{Towards an understanding of the variability of ASHs in $L_{*}$ galaxies}

I use the library just described to assemble $500 \mathrm{ASHs}$, for galaxies that share a virial mass of $M_{200}(z=0)=2 \times 10^{12} M_{\odot}$. I use Monte Carlo generated accretion histories (Fakhouri et al. 2010), 150 of which are displayed in panel a) of Fig. 1, colorcoded by the total accreted stellar mass. Satellite stellar masses are assigned based on a redshift-independent abundance matching relation (Garrison-Kimmel et al. 2014, 0.3 dex 
scatter). Panels $b$ ) and $c$ ) exemplify the assembly of an individual ASH: each accreted and disrupted satellite (color-coded by its accretion redshift) is associated to the spherically averaged density profile of the stars it deposits in the halo. These are retrieved from the library using the relevant time-interval between accretion and $z=0$, and are re-scaled to physical quantities according to the dimensional properties of the merger at hand.

Panel d) displays the spherically averaged density profiles of 150 ASHs built in this manner. At each radius, the halo-to-halo scatter approaches $2 \mathrm{dex}$, and increases at $r \gtrsim 100$, together with an increasing amount of not fully phase-mixed substructure from recent accretion events. Although with a significant scatter, on average, ASHs share a logarithmic density slope $\gamma \sim-2$ within $20 \mathrm{kpc}$, and become steeper with radius, as shown by the dashed guiding lines. The details of this steepening are highly variable: some profiles have marked and sharp breaks, others 'roll' gently towards steeper and steeper slopes, other remain comparatively shallower. The radii where such transitions take place are equally variable. The color-coding in panel $d$ ) indicates the local massweighted satellite-to-host virial mass ratio. On average, the innermost regions of the ASH are contributed by satellites that have larger virial mass ratios at accretion. This gradient has been observed in cosmological hydrodynamical simulations (Rodriguez-Gomez et al. 2016) and I conclude is a direct consequence of dynamical friction (e.g., Amorisco 2015). Color-coding in panel d) reveals that the local mean virial mass ratio also correlates positively with the local density. Panel e) confirms this link by showing a scatter plot of the total accreted stellar mass of the ASH against the "number of main accretion events' $\langle N\rangle$ (i.e. the ratio between the total accreted stellar mass of the ASH and the mean stellar mass of the contributing satellites, mass-weighted by stellar mass itself). The most massive ASHs result from the accretion of just one/two particularly massive satellites, which dominate the ex-situ mass.

Although this technique represents a highly simplified approach, it allows for an efficient exploration of the significant stochasticity of ASHs. Physical ingredients that are neglected here (the host's stellar disk, the morphologies of the accreted dwarfs, any postaccretion star formation etc.) will result in even increased variability. Detailed analysis of a large sample of ASHs concentrating on the systematic correlations that connect density profile and accretion history is the subject of a forthcoming work (Amorisco 2016).

\section{References}

Amorisco, N. C. 2016, in prep.

Amorisco, N. C. 2015, arXiv:1511.08806

Benson, A. J. 2005, MNRAS, 358, 551

Bullock, J. S. \& Johnston, K. V. 2005, ApJ, 635, 931

Cooper, A. P., Cole, S., Frenk, C. S., et al. 2010, MNRAS, 406, 744

Deason, A. J., Belokurov, V., Evans, N. W., \& Johnston, K. V. 2013, ApJ, 763, 113

Fakhouri, O., Ma, C.-P., \& Boylan-Kolchin, M. 2010, MNRAS, 406, 2267

Gao, L., Navarro, J. F., Cole, S., et al. 2008, MNRAS, 387, 536

Garrison-Kimmel, S., Boylan-Kolchin, M., Bullock, J. S., \& Lee, K. 2014, MNRAS, 438, 2578

Gilbert, K. M., Guhathakurta, P., Beaton, R. L., et al. 2012, ApJ, 760, 76

Ibata, R. A., Lewis, G. F., McConnachie, A. W., et al. 2014, ApJ, 780, 128

Jiang, L., Cole, S., Sawala, T., \& Frenk, C. S. 2015, MNRAS, 448, 1674

Ludlow, A. D., Navarro, J. F., Angulo, R. E., et al. 2014, MNRAS, 441, 378

Rodriguez-Gomez, V., Pillepich, A., Sales, L. V., et al. 2016, MNRAS, 458, 2371

Sesar, B., Jurić, M., \& Ivezić, Ž. 2011, ApJ, 731, 4

van Dokkum, P. G., Abraham, R., \& Merritt, A. 2014, ApJL, 782, L24 